\title{
Research on the Influencing Factors of Brand Diffusion via Social Media
}

\author{
Peng Yunan ${ }^{1,2 *}$, Wang Zongshui ${ }^{1,2}$, Zhang Jian ${ }^{1,2}$ \\ ${ }^{1}$ School of Economics and Management, Beijing Information Science and Technology University; \\ ${ }^{2}$ Beijing Key Lab of Green Development and Decision Based on Big Data
}

\begin{abstract}
Purpose] The paper aims to identify the impact factors of brand diffusion via social media. It provides a theoretical reference for enterprises to choose social media and improves brand influence. [Method] First, we summarize the general path of brand diffusion through social media by studying the relevant literatures. Next, four popular social media WeChat, Weibo, Tiktok, and Taobao have been selected as research samples to analyze the different brand diffusion paths. Finally, we summarize the impact of brand diffusion on social media according to the paths. [Results] The result shows that the main factors influencing brand diffusion on social media are diffusion content, information credibility, audience participation, and diffusion path
\end{abstract}

\section{Introduction}

Brand building is an important way to increase the added value of corporate, it is important for enterprises to promote the market share. Enterprises shape brand images through information diffusion and promote a positive relationship between the brand and the public [1]. In traditional brand diffusion, consumers are more contacted to the products. They passively receive brand information and rarely establish communication with companies [2]. Social media allows users to create and share generated content freely based on the internet, so it provides a new way for brand communication. Social media transform brand diffusion from a single way into the multi-way that companies and consumers can communicate freely [3]. Information is interactive on the internet. Thus, the audience can take a variety of channels to spread the information and the role of the audience has changed from the traditional passive "receiver" of information to the "producer" and "disseminator" of the information. Through the interactive forms of search, sharing, comment and praise, the audience makes the brand information spread quickly. Consumers' positive evaluation of the brand makes its image effect improve rapidly. Then "fans" groups appear and they improve the loyalty and identity of consumers to the brand [4]. Besides, the competition between enterprises is more intense now, but social media can provide comprehensive data for enterprises [5]. Social media has been widely used because of its wide range of audiences, accurate positioning of the target audience and low communication cost. The image of the brand determines the public's perception of the brand. There are many influencing factors of brand diffusion in social media. It is meaningful to

\footnotetext{
*Corresponding author: 870431044@qq.com
}

understand brand diffusion mechanisms through social media and improve the brand influence.

\section{Theoretical review}

The brand spreads through communication ${ }^{[6]}$ that connects enterprises and consumers. Brand diffusion can shape brand image and affects consumers' cognition of brand, so it can affect product sales ${ }^{[7]}$. Traditional brand communication mainly relies on marketing, public relations and advertising. The paths can be summarized as brand identification, brand interpretation, brand relationship construction, brand audit, advertising, sponsorship activities, brand official websites ${ }^{[8]}$. It can be seen that the traditional sense of the path is long and may not be able to achieve the planned value-added effect. The media can't be integrated and lack of innovation. The network media has broken the restrictions of information diffusion with the development of the Internet. It promotes the integration of traditional media and network media then forms digital newspapers, touch media, digital broadcasting, etc. It promotes users to search for massive information quickly [9]. Therefore, the path of brand diffusion is constantly changing. Big data can not only add value for the brand diffusion path ${ }^{[10]}$, but also accurate position the differentiated needs of customers. The contact of brand diffusion is more extensive. There are more and more enterprises to pay attention to the content of brand diffusion and the management of the diffusion process.

Social media which based on network media is mainly in the form of WeChat, Weibo, Video, etc. Weibo community, communication community, blog community correspond to the producers, audiences, and organizers of 
information diffusion ${ }^{[11]}$. Social media has changed the way of audiences exchanging and receiving information. It provides new channel for the brand spread. Customers and brand merchants are positive interacted in social media ${ }^{[12]}$, and the way of diffusion and audience differences have an important impact on brand diffusion ${ }^{[13]}$. Social media can divide the target audience into small groups and provide accurate products. So, the audience has become the key impact factor in information diffusion ${ }^{[14]}$. The audiences exchange information through social media, they can search for the needed information anytime and anywhere and make full use of their advantages to make comments on the platform. They are the information sharing and disseminator which makes the information volume of popular brands increase explosively ${ }^{[15]}$. Brand diffusion on social media has advantages of personalized customization, high-speed communication, creativity, etc. It can fully expand the authority of each node in the brand diffusion networks, enhance brand awareness and loyalty of the audience. It has become the main way for brand diffusion. However, brand diffusion in social media is more complex. It is important to deeply understand the brand diffusion path and influencing factors.

\section{Brand diffusion path and influencing factors}

The path of brand diffusion includes strategy determination, elements classification, mode selection, media selection, audience psychological analysis, market communication cycle, globalization and localization, network communication, and effect evaluation ${ }^{[16]}$. Due to the different processes, it can be divided into examining the theme, positioning the target customers, market positioning brand representation, attaching brand culture, determining information, selecting and integrating media, implementing integrated communication, effect evaluation, and regulation, etc. The continuous development of the network promotes the continuous integration of social media, big data, and the Internet. The path of brand diffusion with social media is constantly updated. Brand diffusion is no longer a unilateral "broadcast" communication of brand merchants but becomes a bidirectional (Figure 1).

As shown in Figure 1. On the one hand, the audience finds the needs of brand information and receives the diffusion of brand information on social media. At the same time, the audience's brand preference is screened and matched through the database, then the user is recommended to the brand merchant.

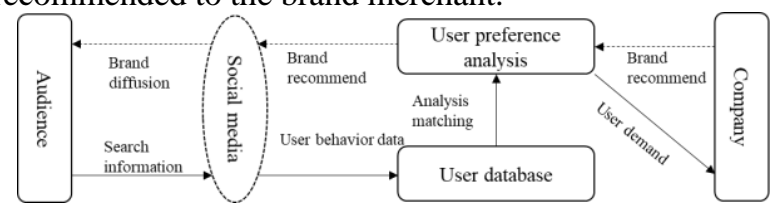

Figure 1. Bidirectional path diffusion in social media

On the other hand, companies share information with customers and choose the target customers according to their preference to carry on the directional brand information dissemination. Brand diffusion links brand merchants and audiences through social media. The audience may not be brand enthusiasts but can become potential consumers by browsing the brand information. For the convenience of social media, more users are using WeChat, Weibo, Tiktok, Taobao. Therefore, taking these four popular social media as the research samples, the differences in diffusion objects, diffusion methods, and diffusion relations has been summarized in Table 1.

TABLE I. BRAND DIFFUSION IN SOCIAL MEDIA

\begin{tabular}{|c|c|c|c|}
\hline Platform & Diffusion Object & Diffusion Method & $\begin{array}{c}\text { Diffusion } \\
\text { Relationship }\end{array}$ \\
\hline Weibo & $\begin{array}{l}\text { Website recommend, } \\
\text { Concerned friends }\end{array}$ & $\begin{array}{l}\text { Following shares, For you, } \\
\text { Weibo hot search, Hot } \\
\text { topic }\end{array}$ & $\begin{array}{l}\text { More } \\
\text { authoritative }\end{array}$ \\
\hline WeChat & Added friends & $\begin{array}{l}\text { Subscriptions recommend, } \\
\text { Friends sharing, Moments, } \\
\text { Advertisements }\end{array}$ & Less authoritative \\
\hline Tiktok & $\begin{array}{l}\text { Recommended or } \\
\text { Concerned friends }\end{array}$ & For you, Following & $\begin{array}{l}\text { More } \\
\text { authoritative }\end{array}$ \\
\hline Taobao & $\begin{array}{l}\text { Friends, Brand } \\
\text { consumers, Brand } \\
\text { followers }\end{array}$ & $\begin{array}{l}\text { Home page recommend, } \\
\text { Friends, sharing }\end{array}$ & Less authoritative \\
\hline
\end{tabular}

Because of the above reasons, we select social platform, short video platform, and e-commerce platform to carry out the following research to further explore their diffusion paths.

\subsection{Brand diffusion of WeChat}

Friends are strong relationships based on the real society in WeChat. Communication in WeChat is based on the permission of friends, which is a kind of "decentralized" equal relationship. WeChat can automatically block 
interference information. Brand diffusion has high trust, strong pertinence, and high interaction stickiness in WeChat. The brand diffusion path in WeChat can be summarized as the sharing of all kinds of channels, personal accounts, Moments, or others. WeChat plays an intermediary role in brand diffusion at this time. The audience's sharing for a brand or asking for help is also the process of brand information diffusion. WeChat becomes the information center because it can change information fluently with QQ, Zhihu, Weibo, etc. The brand followers of WeChat are generally brand-loyal fans and potential customers, which can bring high benefits to the product. First of all, WeChat friends communicate with each other or seek information from friends who are also spreading information. Secondly, the audience receives information when they skim through Moments, Subscriptions, and Mini Programs. At the same time, companies can also advertise at Moments. Also, Subscriptions of WeChat has opened the "Top Stories" function. Friends can click "Top Stories" to share messages among friends after reading the articles. Companies can also select the target audience to push information by data management database. Finally, companies can directly display their preferred brands on the home page according to the user's behavior data through WeChat "shopping" platform (Figure 2).

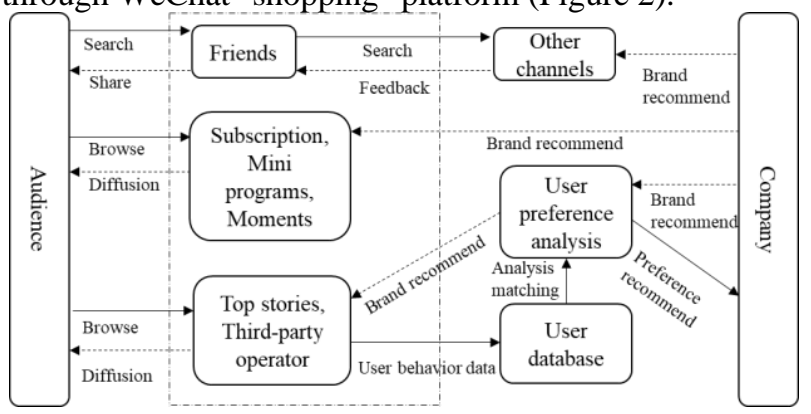

Figure 2. Brand diffusion path of WeChat

There are more detailed descriptions and pictures of products in the subscriptions of WeChat. Friends exist based on the real relationship in WeChat, so brand information can be transmitted to the target audience with higher trust. However, WeChat user data is highly confidential. The user's preference analysis can only be obtained through other related platforms, which makes brand diffusion more difficult.

\subsection{Brand diffusion of Weibo}

Weibo information diffusion has the advantages of low cost, strong connectivity, a high degree of participation, depth of interaction, etc. Enterprises promote their brands by creating hot topics to enhance the influence of brands. Brand diffusion of Weibo has the characteristics of accurate orientation, strong interaction, and community communication ${ }^{[17]}$. Brand diffusion in Weibo is mainly through Internet Celebrities as opinion leaders to deliver brand content in the form of multiple and interesting. Internet Celebrities are more authoritative because of the high popularity of them. The sharing of brand information between friends through chatting accounts for a small part of Weibo. It mainly receives brand publicity through the brand information released by famous bloggers. The brand diffusion released by well-known bloggers may be selfemployed brands or brand merchants who obtain the type of blogger through the data platform and ask them to promote the brand. The audience can freely express their opinions, share or seek information in Weibo. Brand information is more open and audience participation is higher. Besides, the data platform analyzes user preferences through user data and recommends brand information to the audience. The audience can also receive brand information by browsing hot topic information. Secondly, hot topics can be hot searched on Weibo with high hits. Hot search has strong timeliness and focuses on many audiences, which can have a certain social impact. The specific path is shown in Figure 3.

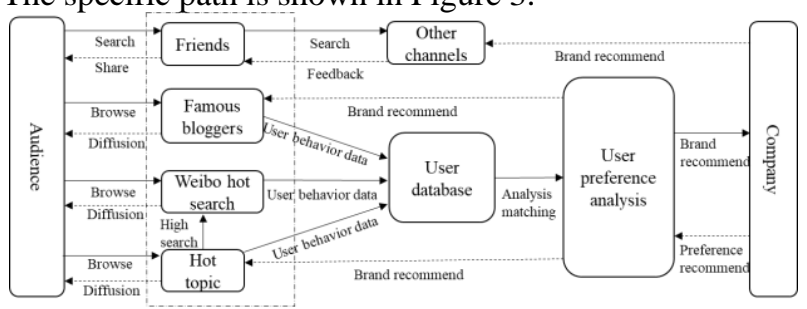

Figure 3. Brand diffusion path of Weibo

Many fans on Weibo are loyal customers or potential users of the brand. Companies can accurately locate the target audience through the data platform. Moreover, Weibo has the functions of crisis public relations and customer management, so it can respond quickly when negative information appears. Weibo is one of the mainstream social platforms that has maintained sustained popularity. It affects all levels of society and becomes an important driving force for public opinion guidance. The information diffusion in Weibo is a weak relationship that has a wide range and fast speed. It brings a huge communication effect to the normalized information dissemination because that popular bloggers have actual authority. However, bloggers can't lead which information to receive. Opinion leaders lead the information release and guide public opinion.

\subsection{Brand diffusion of Tiktok}

Tiktok is a popular platform based on micro-video, which has many advantages such as diversified forms, the low threshold of registration platform ${ }^{[18]}$. Companies advertise through their own or network celebrity Tiktok account. On the one hand, friends can share brand information through the small video. On the other hand, companies can analyze user behavior according to big data and locate user preferences. After that, the data center recommended relevant small videos to the audience. With a high click rate and high comment volume, the micro-video can become a popular online video and bring public opinion influence. The audience receives the brand information while browsing the small video information, as shown in Figure 4. 


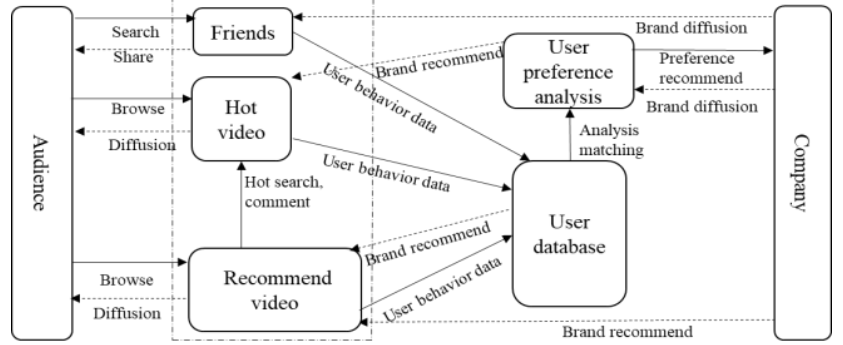

Figure 4. Brand diffusion path of Tiktok

The brand diffusion in Tiktok is more about users' experience after they used products. The content of them is novel and interesting. The audience can have a more intuitive understanding of the product and comment freely, which is more likely to cause impulse consumption. Tiktok is recommended for users to promote videos based on big data and accurately target audiences. But Tiktok is lack of strict review and crisis public relations. There is much false information or bad information in the video. This is easy to cause adverse effects and lose the target audience.

\subsection{Brand diffusion of Taobao}

Taobao is the largest online retail e-commerce social media in Asia. There are more than 500 million users and 268.4 billion transactions during the "Double 11 event" in 2019. As the largest e-commerce retail app, Taobao is the most direct platform for brand information diffusion. Friends can share brand information in Taobao. Taobao platform can also analyze user behavior through daily search based on big data. Then it recommends brand information for users in each functional area of the home page (Figure 5).

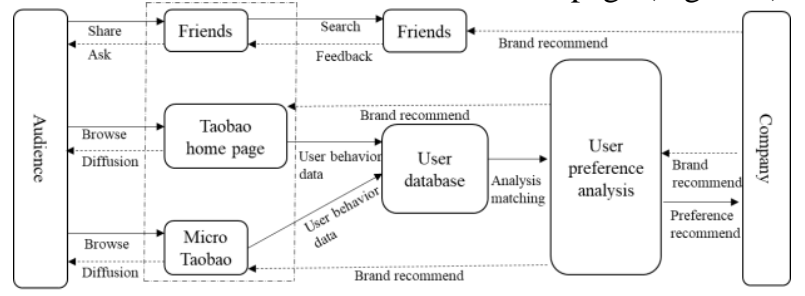

Figure 5. Brand diffusion path of Taobao

\section{Identification of influencing factors of brand diffusion}

According to the above path analysis, the influencing factors of brand diffusion based on social media are mainly content, information credibility, audience participation, brand diffusion path, channel, etc.

In terms of brand diffusion content, the content of diffusion directly affects the audience's cognition of the brand. Enterprises will form their own unique brand culture after the accurate market positioning of their products. The company designs and disseminates the brand. Mass information and differentiated forms in social media make the brand content diversified. The youth are more personalized and differentiated, which requires enterprises to innovate brand diffusion forms and make their content more attractive. Then it constantly meets the audience's personalized psychological needs. The content of brand communication in different social media has different characteristics, which will also affect the audience's choice and positioning of products.

In terms of information sources, the medium is an important link in brand information diffusion. The connectivity of the network makes the brand diffusion of social media compatible with all media information. Media convergence makes brand communication diversified and brings new opportunities and challenges to brand diffusion. Social media advertising is not like traditional TV ads that suddenly disturb the audience. The audience closes or sweeps through the information they don't want to pay attention to, and browses again if they are interested. The platform will also analyze and recommend brand information according to user preferences, which is more selective and personalized. Enterprise official website or network advertisement often relies on social media to be more abundant and diversified. When the information is generated based on the actual friend relationship or based on famous people, the information is more reliable and authoritative. On the contrary, some brand information is lack of authority and trust. The audience will take a neutral attitude. So, the source of information will also affect brand diffusion impact.

In terms of audience participation, the target of brand communication is the audience. The audience shares the brand information equally in social media. The audience's gender, age, education level, and values may cause the audience to choose the brand differently. Their participation in the brand directly affects the brand diffusion effect. The audience can free speech, share, discuss, and comment on their feedback on brand information in social media. Enterprises release brand information on the Internet to guide the audience to actively participate in brand communication which can reduce the audience's rejection. Then the company can accurately position the target audience to attract the audience's attention to brand information. It can not only improve the quality of brand promotion but also bring direct sales. So, different social media should make accurate positioning according to their user groups, and select the target audience through to meet the needs of them

In traditional brand diffusion, information is transmitted to the audience through newspapers and television or other media. But in social media, brand merchants rely on the mutual connection between the media and the audience to form a unique communication mode, effect, information, and feedback information. So, it shortens the distance between the enterprise and the audience, simplifies the path of brand diffusion and the brand information can be transmitted to the audience more efficiently.

\section{Conclusion}

This paper summarizes the general path of brand diffusion in social media and summarizes the more mature brand diffusion path in social media, and compares with it. The results show as follows. Similarities: The content is novel; 
the form is diversified and interesting. Difference: The brand diffusion in WeChat and Weibo is more reliable; it is more powerful in Tiktok; the Taobao brand information is more intuitive and more likely to cause direct consumption; brand diffusion in social media should increase the interest and innovation of its content and meet the differentiated needs of consumers; companies should customize the content of brand communication through different channels; different social media should carry out brand information according to their own audience categories to meet the differentiated needs of the audience; companies should use hot topic in brand promotion in order to find the tipping point of brand diffusion, strengthen the brand's ability of crisis public relations, select authoritative platform for release and avoid adverse impact on the brand; brand diffusion should shorten the path and optimize the communication effect; different channels bring different publicity effects, so companies should choose the appropriate channel according to the product characteristics. The disadvantage of this paper is that only four mature platforms are selected as examples, which are only discussed from the theoretical level. In the future, we will analyze the mechanism of brand communication on social media with objective data.

\section{About the authors}

Peng Yunan is a MA student of the School of Economics and Management, BISTU. Peng Yunan is the corresponding author and can be contacted at 870431044@qq.com.

Wang Zongshui is a Vice Professor of the School of Economics and Management, BISTU.

Zhang Jian is a Professor of the School of Economics and Management, BISTU.

\section{Acknowledgments}

This research was supported by the National Key R\&D Program of China (No. 2017YFB1400500), Beijing Municipal Education Commission Social Science Foundation (No. SM201911232005), and Qinxin Talents Cultivation Program of Beijing Information Science \& Technology University (No. QXTCP B201906).

\section{Reference}

1. $\mathrm{Hu}$ Xiaoyun. Evaluation index of brand communication effect[M]. Beijing: Communication University of China Press,2007.

2. Tang liping. The Situation of Brand Communication in The New Media Environment[J]. Journal of Chinese Youth Social Science,2015(34).

3. Tsimonis G, Dimitriadis S. Brand strategies in social media[J]. Marketing Intelligence\& Planning, 2014 (32).

4. Kaplan, A M, Haenlein, M. Users of the world, unite! The challenges and opportunities of social media[J]. Business Horizons, 2010 (53).
5. Li Fenglin, Liu fang, Yang Wei, etc. Social Media Applications Research Conceptual Framework: Based on Theories, Technologies and Tools[J]. Information Science,2019（37）.

6. Yu Mingyang, Zhu Jida, Xiao Junsong. Brand communication[M]. Shanghai: Shanghai Jiao Tong University Press, 2016.

7. Zhang Cuiling, Wang Qiang. Brand Information Dissemination Methodology in Mobile Internet Era: Evaluation of Brand Communication Theory and Practice[J]. Shanghai Journalism Review,2019(06).

8. Timm F. Wagner, Christian V. Baccarella, Kai-Ingo Voigt. Framing social media communication: Investigating the effects of brand post appeals on user interaction[J]. European Management Journal, 2010(35).

9. Huang Qinghua. Brand Communication Strategy in the Era of Media Convergence[J]. Modern Communication (Journal of Communication University of China), 2016(38).

10. Duan Chunlin, Yan Jimin. Diffusion and Increment: Study on Path Evolution and Value of Brand Communication[J]. Chinese Journal of Journalism \& Communication,2016,(38).

11. Ming Junren, Cao Huizi, Tu Ruide, etc. Research on the Elements and Structure of Social Media Information Ecosystem: Taking the Library Weibo as an Example[J]. Journal of Modern Information, 2019(39).

12. Mar Gómeza, Carmen Lopezb, Arturo Molinaa, An integrated model of social media brand engagement $[\mathrm{J}]$. Computers in Human Behavior,2019(96).

13. Deng En, Xiang Zhiqiang, Huang Yuanji. Analysis on the Influencing Factors of Brand Communication Effect in Internet Financial Industry[J]. Statistics \& Decision,2017(09).

14. Xie Xinzhou, Liu Jinglei, Wang Qiang. Study on the Evaluation of Brand Communication Effect in Social Media[J]. Library and Information Service,2014(58).

15. AHorowitz D M. The new community rules: marketing on the social web[J]. Journal of Product \& Brand Management, 2010 (19).

16. Yang Yuanyuan, Wang Weihong. Research on the Innovation of Enterprise Brand Communication Path in Industry 4.0 Era[J]. Enterprise Economy, 2017(36).

17. Akar E,Topcu B.An examination of the factors influencing consumers' attitudes toward social media marketing[J].Journal of Internet Commerce, 2011(10).

18. Gao Yan. Reflection and Reconstruction of Short Video Marketing Mode in the New Media Era: Taking the Tiktok Short Video Platform as An Example[J]. Viewing on Publishing, 2019(08):62-64. 